\title{
HERMITE SPECTRAL METHODS WITH A TIME-DEPENDENT SCALING FOR PARABOLIC EQUATIONS IN UNBOUNDED DOMAINS*
}

\author{
HEPING MA ${ }^{\dagger}$, WEIWEI SUN ${ }^{\ddagger}$ AND TAO TANG ${ }^{\S}$
}

\begin{abstract}
Hermite spectral methods are investigated for linear diffusion equations and nonlinear convection-diffusion equations in unbounded domains. When the solution domain is unbounded, the diffusion operator no longer has a compact resolvent, which makes the Hermite spectral methods unstable. To overcome this difficulty, a time-dependent scaling factor is employed in the Hermite expansions, which yields a positive bilinear form. As a consequence, stability and spectral convergence can be established for this approach. The present method plays a similar role in the stability of the similarity transformation technique proposed by Funaro and Kavian [Math. Comp., 57 (1991), pp. 597-619]. However, since coordinate transformations are not required, the present approach is more efficient and is easier to implement. In fact, with the time-dependent scaling the resulting discretization system is of the same form as that associated with the classical (straightforward but unstable) Hermite spectral method. Numerical experiments are carried out to support the theoretical stability and convergence results.
\end{abstract}

Key words. Hermite spectral method, time-dependent scaling, stability, convergence

AMS subject classifications. 65N30, 76D99

DOI. $10.1137 / \mathrm{S} 0036142903421278$

1. Introduction. Spectral methods for approximating solutions of differential equations in unbounded domains have received considerable attention, mainly due to their high accuracy and being free from using artificial boundary conditions. The spectral approaches employ orthogonal systems in unbounded domains, e.g., using the Laguerre spectral methods for problems in semibounded or exterior domains $[2$, $4,8,12,17,18,21]$ and the Hermite spectral methods for the problems in unbounded domains $[1,5,6,7,10,20]$. An alternative approximation for such problems is the rational spectral method which has also been studied by several authors $[3,9,11,13$, $25]$.

When the Hermite method is applied to second-order differential equations directly, it is found in [7] that the nonsymmetric bilinear form is not of the desired coercity property. To see this, let us consider the following simple parabolic problem:

* Received by the editors January 13, 2003; accepted for publication (in revised form) April 28, 2004; published electronically April 26, 2005.

http://www.siam.org/journals/sinum/43-1/42127.html

$\dagger$ Department of Mathematics, Shanghai University, Shanghai 200436, China (hpma@mail.shu. edu.cn). The research of this author was supported by the Hong Kong Research Grants Council (project CityU 1084/02P), National Science Foundation of China (project NSFC 10471089), and Special Funds for Major Specialities of Shanghai Education Committee. The major portion of this research was carried out while this author was visiting the City University of Hong Kong.

$¥$ Department of Mathematics, The City University of Hong Kong, Kowloon, Hong Kong (maweiw @math.cityu.edu.hk). The research of this author was supported by the Hong Kong Research Grants Council (project CityU 1084/02P).

$\S$ Department of Mathematics, The Hong Kong Baptist University, Kowloon Tong, Hong Kong, and Institute of Computational Mathematics, The Chinese Academy of Sciences, Beijing 100080, China (ttang@math.hkbu.edu.hk, ttang@lsec.cc.ac.cn). The research of this author was supported by the Hong Kong Research Grants Council (projects HKBU 2044/00P and HKBU2083/01P) and the International Research Team on Complex System of Chinese Academy of Sciences. 


$$
\begin{cases}\partial_{t} U-\nu \partial_{x}^{2} U=f(x, t), & x \in \mathbb{R}, \quad t>0 \\ U(x, 0)=U_{0}(x), & x \in \mathbb{R}\end{cases}
$$

where the diffusion constant $\nu>0$, and $\mathbb{R}=(-\infty, \infty)$. The solution $U$ and its partial derivative $\partial_{x} U$ have to satisfy certain decay conditions as $|x| \rightarrow \infty$. Let $\mathbb{P}_{N}(\mathbb{R})$ be the space of polynomials of degree at most $N$ and let

$$
V_{N}=\left\{v_{N}(x)=\omega_{\beta} \phi_{N}(x) \mid \phi_{N}(x) \in \mathbb{P}_{N}(\mathbb{R})\right\},
$$

where $\omega_{\beta}=\mathrm{e}^{-(\beta x)^{2}}$ with $\beta$ being a constant. The semidiscrete Hermite function method for (1.1) is to find $u_{N}(t) \in V_{N}$ such that for any $\varphi_{N} \in \mathbb{P}_{N}(\mathbb{R})$,

$$
\left\{\begin{array}{l}
\left(\partial_{t} u_{N}(t), \varphi_{N}\right)+\nu\left(\partial_{x} u_{N}(t), \partial_{x} \varphi_{N}\right)=\left(f(t), \varphi_{N}\right), \quad t>0, \\
\left(u_{N}(0), \varphi_{N}\right)=\left(U_{0}, \varphi_{N}\right),
\end{array}\right.
$$

where $(\cdot, \cdot)$ is the conventional inner product in the $L^{2}(\mathbb{R})$ space.

We demonstrate that neither is the nonsymmetric bilinear form in (1.3) coercive nor can a corresponding Gårding's type inequality be established. To show this, we denote by $H_{l}(x)$ the Hermite polynomial of degree $l$ orthogonal on $\mathbb{R}$ with respect to the weight $\omega_{1}(x)=e^{-x^{2}}$. Let $\beta>0$ and let

$$
\underline{H}_{l}(x):=\left(2^{l} l ! \sqrt{\pi}\right)^{-1 / 2} H_{l}(x), \quad H_{l}^{(\beta)}(x):=\sqrt{\beta} \underline{H}_{l}(\beta x) .
$$

Note that $\left\|\underline{H}_{l}\right\|_{\omega_{1}}=1$ and $\left\|H_{l}^{(\beta)}\right\|_{\omega_{\beta}}=1$. Then, for $u_{N}=\omega_{\beta} \phi_{N}$ with $\phi_{N}:=$ $\sum_{l=0}^{N} \hat{u}_{l} H_{l}^{(\beta)}$, we have

$$
\begin{aligned}
\left(\partial_{x} u_{N}, \partial_{x} \phi_{N}\right) & =\left|\phi_{N}\right|_{1, \omega_{\beta}}^{2}+\beta^{2}\left\|\phi_{N}\right\|_{\omega_{\beta}}^{2}-2 \beta^{4}\left\|x \phi_{N}\right\|_{\omega_{\beta}}^{2} \\
& =-2 \beta^{2} \sum_{l=2}^{N} \sqrt{l(l-2)} \hat{u}_{l} \hat{u}_{l-2},
\end{aligned}
$$

which cannot be controlled by $\left\|u_{N}\right\|_{\omega_{\beta}^{-1}}^{2}=\sum_{l=0}^{N}\left|\hat{u}_{l}\right|^{2}$. In other words, the stability for (1.3) cannot be established by using the classical energy method. On the other hand, the instability is observed numerically, as seen in section 6 . To overcome this difficulty, a similarity transformation was introduced by Funaro and Kavian [6], which is defined by

$$
s=\ln (1+t), \quad y=x(1+t)^{-\frac{1}{2}} .
$$

With this transformation, they were able to obtain the optimal error estimate of the Hermite function approximation for the linear problem (1.1). This similarity transformation technique has been extended recently to study the nonlinear convectiondiffusion equations; see, e.g., $[7,10]$. By using this transformation, the diffusion operator in (1.1) is changed into an operator whose eigenfunctions are the Hermite functions. This property can lead to a desired stability result. However, the transformation may make the underlying equations more complicated, which leads to difficulties in theoretical analysis and practical implementation. It is desirable to develop some simpler and more efficient Hermite spectral methods.

In this paper, we present a Petrov-Galerkin Hermite spectral method which uses a time-dependent weight function. On the one hand, the method keeps the advantage of 
the similarity transformation method, namely, it gives a positive definite bilinear form. On the other hand, the scheme can be easily formulated in the classical form of (1.3), without introducing any extra new terms. As a result, a priori explicit transformation is not needed. Moreover, the time-dependent weight function behaves like a spatial scaling. The importance of the scaling factor has been demonstrated by Tang [22] and Schumer and Holloway [20]. We will apply the proposed method to the analysis of the nonlinear convection-diffusion equations. Stability and optimal error estimates for the Hermite spectral methods, in both semidiscrete and fully discrete forms, are obtained for the nonlinear equation. It will be shown by numerical experiments that the timedependent weight works well for solutions with time-dependent and time-independent decays.

An outline of the paper is as follows. In section 2 we briefly discuss the Hermite spectral methods with a time-dependent scaling. Section 3 presents some basic properties of the Hermite functions in weighted spaces, which will be useful in the stability and convergence analysis. In sections 4 and 5 , stability and convergence analysis is carried out for the semidiscrete and fully discrete schemes, respectively. The analysis is devoted not only to the linear parabolic equation (1.1), but also to the nonlinear convection-diffusion problems. In section 6 , numerical results will be presented.

2. Hermite method with time-dependent scaling. We present a PetrovGalerkin Hermite spectral method with a time-dependent scaling for the simple model problem (1.1). Let $\alpha=\alpha(t)>0$. We take

$$
\alpha(t)=\frac{1}{2 \sqrt{\nu \delta_{0}(\delta t+1)}},
$$

where $\delta_{0}$ and $\delta$ are some positive parameters. It can be verified that

$$
\alpha^{\prime}(t)=-2 \nu \delta_{0} \delta \alpha^{3(t)} .
$$

The motivation for this choice of $\alpha$ can be found in Remark 4.1 in section 4 . The semidiscrete Hermite spectral method for (1.1) is to find $u_{N}(t) \in V_{N}(t)$ such that for any $\varphi_{N} \in \mathbb{P}_{N}(\mathbb{R})$,

$$
\left\{\begin{array}{l}
\left(\partial_{t} u_{N}(t), \varphi_{N}\right)+\nu\left(\partial_{x} u_{N}(t), \partial_{x} \varphi_{N}\right)=\left(f(t), \varphi_{N}\right), \quad t>0 \\
\left(u_{N}(0), \varphi_{N}\right)=\left(U_{0}, \varphi_{N}\right)
\end{array}\right.
$$

where the trial space $V_{N}(t)$ is defined by

$$
V_{N}(t)=\left\{v_{N}(x)=\omega_{\alpha(t)} \phi_{N}(x) \mid \phi_{N}(x) \in \mathbb{P}_{N}(\mathbb{R})\right\} .
$$

The scheme (2.2) is almost the same as (1.3): the only difference is that here the weight function $\omega_{\alpha}$ in the trial function space $V_{N}$ varies with time. The scheme (2.2) can be rewritten as

$$
\frac{d}{d t}\left(u_{N}(t), \varphi_{N}(t)\right)+\left(u_{N}(t), L^{*} \varphi_{N}(t)\right)=\left(f(t), \varphi_{N}(t)\right),
$$

where $L^{*}:=-\partial_{t}-\nu \partial_{x}^{2}$. To simplify the computation, let

$$
u_{N}(x, t)=\frac{\omega_{\alpha}}{\sqrt{\pi}} \sum_{l=0}^{N} \hat{u}_{l}(t) H_{l}(\alpha x), \quad \varphi_{N}(x, t)=\frac{\alpha(t)}{\left(2^{m} m !\right)} H_{m}(\alpha(t) x) \quad(0 \leq m \leq N) .
$$


In other words, we expand the unknown solution using the scaled Hermite functions with a time-dependent scaling factor. The test function $\varphi_{N}$ is now also dependent on $t$. It can be verified that

$$
\begin{aligned}
& \left(\omega_{\alpha} H_{l}(\alpha x), L^{*}\left(\alpha H_{m}(\alpha x)\right)\right) \\
& =-\alpha^{\prime} \alpha^{-1}\left(\left\|H_{m}\right\|_{\omega_{1}}^{2} \delta_{l m}+\left(y H_{l}, H_{m}^{\prime}\right)_{\omega_{1}}\right)-2 \nu \alpha^{2}\left(y H_{l}, H_{m}^{\prime}\right)_{\omega_{1}}+\nu \alpha^{2}\left\|H_{m}^{\prime}\right\|_{\omega_{1}}^{2} \delta_{l m} \\
& =\delta_{0} \delta \nu \alpha^{2}\left\|H_{m}\right\|_{\omega_{1}}^{2} \delta_{l m}+\left(\delta_{0} \delta-1\right) \nu \alpha^{2}\left(H_{l+1}+2 l H_{l-1}, 2 m H_{m-1}\right)_{\omega_{1}}+\nu \alpha^{2}\left|H_{m}\right|_{1, \omega_{1}}^{2} \delta_{l m} \\
& =\nu \alpha^{2} 2^{m} m ! \sqrt{\pi}\left(2 \delta_{0} \delta \delta_{l m}+\left(\delta_{0} \delta-1\right)\left(\delta_{(l+2) m}+2 m \delta_{l m}\right)+2 m \delta_{l m}\right) .
\end{aligned}
$$

Applying the above result to (2.2) gives

$$
\left\{\begin{array}{l}
\frac{d \mathbf{u}(t)}{d t}+\nu \alpha(t)^{2} \mathbf{A} \mathbf{u}(t)=\mathbf{f}(t), \quad t>0 \\
(\mathbf{u}(0))_{m}=\alpha(0)\left(2^{m} m !\right)^{-1}\left(U_{0}, H_{m}(\alpha(0) x)\right), \quad 0 \leq m \leq N
\end{array}\right.
$$

where $\alpha(0)=1 / 2 \sqrt{\nu \delta_{0}}, \mathbf{u}=\left(\hat{u}_{0}, \hat{u}_{1}, \ldots, \hat{u}_{N}\right)^{T}$. The elements of the matrix A are given by

$$
(\mathbf{A})_{m l}= \begin{cases}2(m+1) \delta_{0} \delta, & l=m \\ \delta_{0} \delta-1, & l=m-2, \\ 0 & \text { otherwise, } 0 \leq l, m \leq N\end{cases}
$$

and the entries $f_{m}$ of $\mathbf{f}$ are given by

$$
\begin{aligned}
\hat{f}_{m}:=(\mathbf{f})_{m} & =\alpha\left(2^{m} m !\right)^{-1}\left(f, H_{m}(\alpha x)\right) \\
& =\left(2^{m} m !\right)^{-1}\left(\mathrm{e}^{y^{2}} f\left(\alpha^{-1} y\right), H_{m}(y)\right)_{\omega_{1}} .
\end{aligned}
$$

Fully discrete methods can be designed by using (2.6) based on the method-of-lines approach. Here we consider the Crank-Nicolson scheme. Let $\tau$ be the time-step $t_{k}=k \tau\left(k=0,1, \ldots, n_{T} ; T=n_{T} \tau\right)$, and let $\mathbf{v}^{k}=\mathbf{v}\left(t_{k}\right)$. The fully discrete PetrovGalerkin method for (1.1) is to find

$$
u_{N}^{k}=\frac{\omega_{\alpha\left(t_{k}\right)}}{\sqrt{\pi}} \sum_{l=0}^{N} \hat{u}_{l}^{k} H_{l}\left(\alpha\left(t_{k}\right) x\right)
$$

such that

$$
\begin{cases}\frac{\mathbf{u}^{k+1}-\mathbf{u}^{k}}{\tau}+\nu \alpha^{2}\left(t_{k}+\tau / 2\right) \mathbf{A} \frac{\mathbf{u}^{k+1}+\mathbf{u}^{k}}{2}=\frac{\mathbf{f}^{k+1}+\mathbf{f}^{k}}{2}, & 0 \leq k \leq n_{T}-1 \\ \left(\mathbf{u}^{0}\right)_{m}=\left(2^{m} m !\right)^{-1}\left(\mathrm{e}^{y^{2}} U_{0}(y / \alpha(0)), H_{m}(y)\right)_{\omega_{1}}, & 0 \leq m \leq N\end{cases}
$$

Since the matrix $\mathbf{A}$ is independent of time, the above scheme can be solved easily.

Remark 2.1. Note that the matrix $\mathbf{A}$ is an upper triangular matrix whose diagonal entries are $2(m+1) \delta_{0} \delta$. By the classical stability theory, both the semidiscrete scheme (2.6) and fully discrete scheme (2.7) are stable and convergent provided that $\delta_{0} \delta>0$. However, $\delta_{0}=0$ in the classical approach (1.3) yields numerical instability. 
3. Approximation properties of Hermite functions. In this section, we present some basic approximation properties for the Hermite functions and the Hermite polynomials. Some of them are similar to those obtained in $[5,6,7,19,23,24]$ and we will only briefly outline the proofs.

Let $H^{\sigma}(\mathbb{R}):=W^{\sigma, 2}(\mathbb{R})$ be the Sobolev spaces with the norm $\|\cdot\|_{\sigma}$ and seminorm $|\cdot|_{\sigma}$. For a nonnegative weight $\omega(x)$ on $\mathbb{R}$, the inner product and norm of $L_{\omega}^{2}(\mathbb{R})$ are denoted by $(\cdot, \cdot)_{\omega}$ and $\|\cdot\|_{\omega}$, respectively. The subscript $\omega$ will be dropped whenever $\omega(x) \equiv 1$. For a positive integer $\sigma$, the weighted Sobolev space $H_{\omega}^{\sigma}(\mathbb{R})$ is defined by

$$
H_{\omega}^{\sigma}(\mathbb{R})=\left\{v \mid \partial_{x}^{r} v \in L_{\omega}^{2}(\mathbb{R}), \quad 0 \leq r \leq \sigma\right\}
$$

with the seminorm and norm

$$
|v|_{\sigma, \omega}=\left\|\partial_{x}^{\sigma} v\right\|_{\omega}, \quad\|v\|_{\sigma, \omega}=\left(\sum_{r=0}^{\sigma}|v|_{r, \omega}^{2}\right)^{1 / 2} .
$$

Denote by $H_{l}(x)$ the Hermite polynomial of degree $l$ :

$$
H_{l}(x)=(-1)^{l} \omega_{1}^{-1}(x) \partial_{x}^{l}\left(\omega_{1}(x)\right) .
$$

In theoretical analysis, it seems more convenient to use the normalized Hermite polynomials

$$
\underline{H}_{l}(x):=\left(2^{l} l ! \sqrt{\pi}\right)^{-1 / 2} H_{l}(x) .
$$

We will work with the scaled Hermite polynomial $H_{l}^{(\beta)}(x):=\sqrt{\beta} \underline{H}_{l}(\beta x)$, where $\beta>0$ is a constant. For nonnegative integers $r$ and $l$, let

$$
A_{l}^{r}= \begin{cases}l ! /(l-r) !, & l \geq r \geq 1, \\ 1, & l \geq 0, r=0, \\ 0, & l<r .\end{cases}
$$

We have

$$
\left(\partial_{x}^{r} H_{l}^{(\beta)}, \partial_{x}^{r} H_{m}^{(\beta)}\right)_{\omega_{\beta}}=\beta^{2 r}\left(\partial_{x}^{r} \underline{H}_{l}, \partial_{x}^{r} \underline{H}_{m}\right)_{\omega_{1}}=\left(2 \beta^{2}\right)^{r} \sqrt{A_{l}^{r} A_{m}^{r}} \delta_{l m}
$$

so that $\left\{\partial_{x}^{r} H_{l}^{(\beta)}\right\}$ are orthogonal on $\mathbb{R}$ with respect to the weight $\omega_{\beta}=e^{-(\beta x)^{2}}$. Let $P_{N}^{\beta}: L_{\omega_{\beta}}^{2}(\mathbb{R}) \rightarrow \mathbb{P}_{N}(\mathbb{R})$ be the $L_{\omega_{\beta}}^{2}$-orthogonal projection operator defined by

$$
\left(P_{N}^{\beta} v-v, \varphi_{N}\right)_{\omega_{\beta}}=0 \quad \forall \varphi_{N} \in \mathbb{P}_{N}(\mathbb{R}) .
$$

For $v \in H_{\omega_{\beta}}^{r}(\mathbb{R}) \quad(r<N)$, we have $\partial_{x}^{r} P_{N}^{\beta} v=P_{N-r}^{\beta} \partial_{x}^{r} v$ and

$$
\left(\partial_{x}^{r}\left(P_{N}^{\beta} v-v\right), \varphi_{N-r}\right)_{\omega_{\beta}}=0 \quad \forall \varphi_{N-r} \in \mathbb{P}_{N-r}(\mathbb{R}) .
$$

We consider the approximation by the Hermite functions; i.e., we approximate $v \omega_{\beta}^{-1}$ by using the Hermite polynomials. Let $\mathcal{P}_{N}^{\beta}: L_{\omega_{\beta}^{-1}}^{2}(\mathbb{R}) \rightarrow V_{N}$ be the $L_{\omega_{\beta}^{-1}}^{2}$ orthogonal projection operator defined by

$$
\left(\mathcal{P}_{N}^{\beta} v-v, \varphi_{N}\right)_{\omega_{\beta}^{-1}}=0 \quad \forall \varphi_{N} \in V_{N} .
$$


It is easy to verify that $\mathcal{P}_{N}^{\beta} v=\omega_{\beta} P_{N}^{\beta}\left(v \omega_{\beta}^{-1}\right)$. For $v \in H_{\omega_{\beta}^{-1}}^{r}(\mathbb{R})(r<N)$, we have $\partial_{x}^{r} \mathcal{P}_{N}^{\beta} v=\mathcal{P}_{N+r}^{\beta} \partial_{x}^{r} v$ and

$$
\left(\partial_{x}^{r}\left(\mathcal{P}_{N}^{\beta} v-v\right), \varphi_{N+r}\right)=0 \quad \forall \varphi_{N+r} \in \mathbb{P}_{N+r}(\mathbb{R}) .
$$

LEMMA 3.1. If $r$ is a nonnegative integer, then $v \in H_{\omega_{\beta}^{-1}}^{r}(\mathbb{R})$ is equivalent to $v \omega_{\beta}^{-1} \in H_{\omega_{\beta}}^{r}(\mathbb{R})$. Moreover,

$$
\begin{aligned}
& \sum_{j=0}^{r}\left(2 \beta^{2}\right)^{r-j}\left\|\partial_{x}^{j}\left[\left(I-P_{m}^{\beta}\right)\left(v \omega_{\beta}^{-1}\right)\right]\right\|_{\omega_{\beta}}^{2} \leq\left\|\partial_{x}^{r}\left[\left(I-\mathcal{P}_{m}^{\beta}\right) v\right]\right\|_{\omega_{\beta}^{-1}}^{2} \quad \forall m \geq 0, \\
& \left\|\partial_{x}^{r}\left[\left(I-\mathcal{P}_{N}^{\beta}\right) v\right]\right\|_{\omega_{\beta}^{-1}} \leq C(r)\left\|\partial_{x}^{r}\left[\left(I-P_{N}^{\beta}\right)\left(v \omega_{\beta}^{-1}\right)\right]\right\|_{\omega_{\beta}} \quad \forall N>r,
\end{aligned}
$$

where $P_{0}^{\beta}=\mathcal{P}_{0}^{\beta}=\mathbf{0}$ and $C(r)$ is a constant depending only on $r$.

Proof. By a direct calculation,

$$
\partial_{x}^{r}\left(\omega_{\beta} H_{l}^{(\beta)}(x)\right)=(-\beta)^{r} 2^{r / 2} \sqrt{A_{l+r}^{r}} \omega_{\beta} H_{l+r}^{(\beta)}(x) .
$$

Using this result we can verify that $\left\{\partial_{x}^{r}\left(\omega_{\beta} H_{l}^{(\beta)}\right)\right\}$ are orthogonal with respect to the weight $\omega_{\beta}^{-1}$ on $\mathbb{R}$ :

$$
\begin{aligned}
\left(\partial_{x}^{r}\left(\omega_{\beta} H_{l}^{(\beta)}\right), \partial_{x}^{r}\left(\omega_{\beta} H_{m}^{(\beta)}\right)\right)_{\omega_{\beta}^{-1}} & =\left(2 \beta^{2}\right)^{r} \sqrt{A_{l+r}^{r} A_{m+r}^{r}}\left(H_{l+r}^{(\beta)}, H_{m+r}^{(\beta)}\right)_{\omega_{\beta}} \\
& =\left(2 \beta^{2}\right)^{r} \sqrt{A_{l+r}^{r} A_{m+r}^{r}} \delta_{l m} \quad \forall l, m \geq r \geq 0 .
\end{aligned}
$$

Let $v=\omega_{\beta} \sum_{l=0}^{\infty} \hat{v}_{l} H_{l}^{(\beta)}$. Then we have

$$
\left(I-\mathcal{P}_{m}^{\beta}\right) v=\omega_{\beta}\left(I-P_{m}^{\beta}\right)\left(v \omega_{\beta}^{-1}\right)=\omega_{\beta} \sum_{l \geq m} \hat{v}_{l} H_{l}^{(\beta)} .
$$

The above result, together with (3.9), gives

$$
\begin{aligned}
& \left\|\partial_{x}^{r}\left[\left(I-\mathcal{P}_{m}^{\beta}\right) v\right]\right\|_{\omega_{\beta}^{-1}}^{2}=\left(2 \beta^{2}\right)^{r} \sum_{l \geq m} A_{l+r}^{r}\left|\hat{v}_{l}\right|^{2} \\
& \geq\left(2 \beta^{2}\right)^{r} \sum_{l \geq m} \sum_{j=0}^{r} A_{l}^{j}\left|\hat{v}_{l}\right|^{2} \geq\left(2 \beta^{2}\right)^{r} \sum_{j=0}^{r} \sum_{l \geq \max \{m, j\}} A_{l}^{j}\left|\hat{v}_{l}\right|^{2} \\
& \quad=\sum_{j=0}^{r}\left(2 \beta^{2}\right)^{r-j}\left\|\partial_{x}^{j}\left[\left(I-P_{m}^{\beta}\right)\left(v \omega_{\beta}^{-1}\right)\right]\right\|_{\omega_{\beta}}^{2} .
\end{aligned}
$$

This proves the result (3.6). The inequality (3.7) can be established similarly.

Lemma 3.2. If $0 \leq r \leq \sigma<N$, then

$$
\begin{array}{ll}
\left\|\partial_{x}^{r}\left(v-P_{N}^{\beta} v\right)\right\|_{\omega_{\beta}} \leq C(r, \sigma)\left(2 \beta^{2} N\right)^{(r-\sigma) / 2}\left\|\partial_{x}^{\sigma} v\right\|_{\omega_{\beta}} & \forall v \in H_{\omega_{\beta}}^{\sigma}(\mathbb{R}), \\
\left\|\partial_{x}^{r}\left(v-\mathcal{P}_{N}^{\beta} v\right)\right\|_{\omega_{\beta}^{-1}} \leq C(r, \sigma)\left(2 \beta^{2} N\right)^{(r-\sigma) / 2}\left\|\partial_{x}^{\sigma} v\right\|_{\omega_{\beta}^{-1}} & \forall v \in H_{\omega_{\beta}^{-1}}^{\sigma}(\mathbb{R}),
\end{array}
$$

where $C(r, \sigma)$ is a constant depending only on $r$ and $\sigma$. 
Proof. Let $v=\sum_{l=0}^{\infty} \hat{v}_{l} H_{l}^{(\beta)}$. Then, it follows from (3.1) that

$$
\begin{aligned}
& \left\|\partial_{x}^{r}\left(v-P_{N}^{\beta} v\right)\right\|_{\omega_{\beta}}^{2}=\sum_{l>N}\left(2 \beta^{2}\right)^{r} A_{l}^{r}\left|\hat{v}_{l}\right|^{2} \\
& \quad=\left(2 \beta^{2}\right)^{r-\sigma} \sum_{l>N}\left(A_{l-r}^{\sigma-r}\right)^{-1}\left(2 \beta^{2}\right)^{\sigma} A_{l}^{\sigma}\left|\hat{v}_{l}\right|^{2} \leq\left(2 \beta^{2}\right)^{r-\sigma}\left(A_{N+1-r}^{\sigma-r}\right)^{-1}\left\|\partial_{x}^{\sigma}\left(v-P_{N}^{\beta} v\right)\right\|_{\omega_{\beta}}^{2} \\
& \quad=\left(2 \beta^{2}\right)^{r-\sigma} \prod_{m=r-1}^{\sigma-2}\left(1-\frac{m}{N}\right)^{-1} N^{r-\sigma}\left\|\partial_{x}^{\sigma}\left(v-P_{N}^{\beta} v\right)\right\|_{\omega_{\beta}}^{2},
\end{aligned}
$$

which gives (3.10). Using (3.7), (3.10), and (3.6) gives

$$
\begin{aligned}
& \left\|\partial_{x}^{r}\left(v-\mathcal{P}_{N}^{\beta} v\right)\right\|_{\omega_{\beta}^{-1}}^{2} \leq C(r)\left\|\partial_{x}^{r}\left[\left(I-P_{N}^{\beta}\right)\left(v \omega_{\beta}^{-1}\right)\right]\right\|_{\omega_{\beta}} \\
& \quad \leq C(r, \sigma)\left(2 \beta^{2} N\right)^{(r-\sigma) / 2}\left\|\partial_{x}^{\sigma}\left[\left(I-P_{N}^{\beta}\right)\left(v \omega_{\beta}^{-1}\right)\right]\right\|_{\omega_{\beta}} \\
& \quad \leq C(r, \sigma)\left(2 \beta^{2} N\right)^{(r-\sigma) / 2}\left\|\partial_{x}^{\sigma}\left[\left(I-\mathcal{P}_{N}^{\beta}\right) v\right]\right\|_{\omega_{\beta}^{-1}},
\end{aligned}
$$

which gives (3.11).

LEMma 3.3. Let $r, \sigma$ be nonnegative integers. We have

$$
\begin{array}{ll}
\lim _{|x| \rightarrow \infty} x\left(\partial_{x}^{r} v\right)^{2}(x) \omega_{\beta}^{-1}(x) \rightarrow 0 & \forall v \in H_{\omega_{\beta}^{-1}}^{\sigma}(\mathbb{R}), r \leq \sigma-1, \\
\left\|v^{2} \omega_{\beta}^{-1}\right\|_{L^{\infty}(\mathbb{R})} \leq 2|v|_{1, \omega_{\beta}^{-1}}\|v\|_{\omega_{\alpha}^{-1}} & \forall v \in H_{\omega_{\beta}^{-1}}^{1}(\mathbb{R}), \\
\left|\varphi_{N}\right|_{\sigma, \omega_{\beta}^{-1}} \leq\left(4 \beta^{2} N\right)^{(\sigma-r) / 2}\left|\varphi_{N}\right|_{r, \omega_{\beta}^{-1}} & \forall \varphi_{N} \in V_{N}, r \leq \sigma \leq N, \\
\left\|\sqrt{\omega_{\beta}^{-1}} \varphi_{N}\right\|_{L^{\infty}(\mathbb{R})} \leq 2\left(\beta^{2} N\right)^{1 / 4}\left\|\varphi_{N}\right\|_{\omega_{\beta}^{-1}} & \forall \varphi_{N} \in V_{N}, r \leq \sigma \leq N .
\end{array}
$$

Proof. The first two results, (3.12) and (3.13), can be obtained by the arguments similar to those given in $[5,7]$. Let $\varphi_{N}=\omega_{\beta} \sum_{l=0}^{N} \hat{\varphi}_{l} H_{l}^{(\beta)} \in V_{N}$. It follows from (3.9) that

$$
\begin{aligned}
\left|\varphi_{N}\right|_{\sigma, \omega_{\beta}^{-1}}^{2} & =\left(2 \beta^{2}\right)^{\sigma-r}\left(2 \beta^{2}\right)^{r} \sum_{l=0}^{N} A_{l+\sigma}^{\sigma-r} A_{l+r}^{r}\left|\hat{\varphi}_{l}\right|^{2} \\
& \leq\left(2 \beta^{2} N\right)^{\sigma-r} \prod_{j=r+1}^{\sigma}\left(1+\frac{j}{N}\right)\left|\varphi_{N}\right|_{r, \omega_{\beta}^{-1}}^{2}
\end{aligned}
$$

which gives (3.14). Moreover, using (3.13) and (3.14) gives

$$
\left\|\omega_{\beta}^{-1} \varphi_{N}^{2}\right\|_{L^{\infty}(\mathbb{R})} \leq 2\left(4 \beta^{2} N\right)^{1 / 2}\left\|\varphi_{N}\right\|_{\omega_{\beta}^{-1}}^{2} \leq 4 \beta N^{1 / 2}\left\|\varphi_{N}\right\|_{\omega_{\beta}^{-1}}^{2} .
$$

This completes the proof of this lemma.

4. Stability and convergence: Semidiscretization. To demonstrate the stability and convergence analysis for the proposed spectral method, we take the timedependent weight

$$
\omega_{\alpha(t)}=\mathrm{e}^{-(\alpha(t) x)^{2}}
$$


where $\alpha(t)$ is defined by $(2.1)$. We expand

$$
u_{N}(x, t)=\omega_{\alpha(t)} \sum_{l=0}^{N} \hat{u}_{l}(t) H_{l}^{(\alpha(t))}(x) .
$$

It can be verified that $\left\|u_{N}\right\|_{\omega_{\alpha}^{-1}}=\|\mathbf{u}\|$. The solution expansion (4.2) is slightly different from the one in (2.5) but is more suitable for theoretical analysis. With this expansion, the matrix form for the scheme (2.2) becomes

$$
\frac{d \mathbf{u}}{d t}+\nu \alpha(t)^{2} \mathbf{B u}=\mathbf{f},
$$

where the elements of the matrices $\mathbf{B}$ and the vector $\mathbf{f}$ are given by

$$
\begin{aligned}
& \text { (B) })_{m l}= \begin{cases}\delta_{0} \delta(2 m+1), & l=m, \\
\left(\delta_{0} \delta-1\right) 2 \sqrt{m(m-1)}, & l=m-2, \\
0 & \text { otherwise }\end{cases} \\
& \hat{f}_{m}:=(\mathbf{f})_{m}=\left(f, H_{m}^{(\alpha)}\right), \quad 0 \leq l, m \leq N .
\end{aligned}
$$

The stability and convergence properties can be established following the discussions in section 2. To be more precise, let

$$
\underline{\delta}=\min \left\{1,2 \delta_{0} \delta-1\right\}>0, \quad \mathbf{D}=2 \operatorname{diag}(0,1, \ldots, N)
$$

and let $\mathbf{I}$ be the identity matrix. Since

$$
\mathbf{u}^{T} \mathbf{B u} \geq \underline{\delta} \mathbf{u}^{T}(\mathbf{D}+\mathbf{I}) \mathbf{u},
$$

we obtain

$$
\begin{aligned}
& \|\mathbf{u}(t)\|^{2}+\underline{\delta} \nu \int_{0}^{t} \alpha^{2}\left\|(\mathbf{D}+\mathbf{I})^{1 / 2} \mathbf{u}(s)\right\|^{2} d s \\
& \quad \leq\|\mathbf{u}(0)\|^{2}+4 \delta_{0} \underline{\delta}^{-1} \int_{0}^{t}(\delta s+1)\left\|(\mathbf{D}+\mathbf{I})^{-1 / 2} \mathbf{f}(s)\right\|^{2} d s, \quad t>0
\end{aligned}
$$

or, equivalently,

$$
\begin{aligned}
& \left\|u_{N}(t)\right\|_{\omega_{\alpha}^{-1}}^{2}+\underline{\delta} \nu \int_{0}^{t}\left|u_{N}(t)\right|_{1, \omega_{\alpha}^{-1}}^{2} d s \\
& \quad \leq\left\|u_{N}(0)\right\|_{\omega_{\alpha}^{-1}}^{2}+(\underline{\delta} \nu)^{-1} \int_{0}^{t}\left\|\partial_{x}^{-1} f(s)\right\|_{\omega_{\alpha}^{-1}}^{2} d s
\end{aligned}
$$

where $\partial_{x}^{-1} v(x)=\int_{-\infty}^{x} v(y) d y$.

Remark 4.1. In the classical approach (1.3), we fail to obtain the stability due to the term $\left\|x \phi_{N}\right\|_{\omega_{\beta}}$ in (1.4). However, when $\alpha$ depends on time, an extra term is gained in the $\left\|x u_{N}\right\|_{\omega_{\alpha}}$ term:

$$
\begin{aligned}
& \frac{d}{d t}\left\|u_{N}(t)\right\|_{\omega_{\alpha}^{-1}}^{2}+2 \nu\left(\left|u_{N}\right|_{1, \omega_{\alpha}^{-1}}^{2}-\alpha^{2}\left\|u_{N}(t)\right\|_{\omega_{\alpha}^{-1}}^{2}\right) \\
& \quad-2 \alpha\left(\alpha^{\prime}+2 \nu \alpha^{3}\right)\left\|x u_{N}(t)\right\|_{\omega_{\alpha}^{-1}}^{2}=2\left(f(t), u_{N}(t)\right)_{\omega_{\alpha}^{-1}} .
\end{aligned}
$$

Stability can be obtained if $\alpha$ is chosen to satisfy $\alpha^{\prime}+2 \nu \alpha^{3} \leq 0$. 
We now briefly outline the convergence of the approximation (2.2). Our rigorous analysis will be carried out for the nonlinear convection-diffusion equations, which take (2.2) as a special case. It is interesting to note that the solutions of (1.3) and (2.2) are both of the same form: $u_{N}=\mathcal{P}_{N}^{\alpha} U$. In fact, assuming $U \in C\left(0, T ; H_{\omega_{\alpha}}^{1}(\mathbb{R})\right)$, we have from (3.5) that for any $\varphi_{N} \in \mathbb{P}_{N}(\mathbb{R})$,

$\left\{\begin{array}{l}\left(\partial_{t} \mathcal{P}_{N}^{\alpha} U(t), \varphi_{N}\right)+\nu\left(\partial_{x} \mathcal{P}_{N}^{\alpha} U(t), \partial_{x} \varphi_{N}\right)=\left(\partial_{t} U(t), \varphi_{N}\right)+\nu\left(\partial_{x} U(t), \partial_{x} \varphi_{N}\right)=\left(f(t), \varphi_{N}\right), \\ \left(\mathcal{P}_{N}^{\alpha} U(0), \varphi_{N}\right)=\left(U_{0}, \varphi_{N}\right) .\end{array}\right.$

However, the scheme (1.3) may not work since the bilinear form is not coercive. Since $u_{N}=\mathcal{P}_{N}^{\alpha} U$, it follows from (3.11) that if $U \in C\left(0, T ; H_{\omega_{\alpha}^{-1}}^{\sigma}(\mathbb{R})\right)(\sigma \geq 1)$, then

$$
\left\|u_{N}(t)-U(t)\right\|_{r, \omega_{\alpha}^{-1}} \leq C N^{(r-\sigma) / 2}\|U(t)\|_{\sigma, \omega_{\alpha}^{-1}} \quad \forall 0 \leq r \leq \sigma, \quad t \in(0, T),
$$

which is analogous to the result obtained in [6] by using the similarity transformation.

The above method can be easily applied to some nonlinear equations. Consider the nonlinear convection-diffusion equation

$$
\begin{cases}\partial_{t} U+\partial_{x} F(U)-\nu \partial_{x}^{2} U=f(x, t), & (x, t) \in \mathbb{R} \times(0, T), \\ U(x, 0)=U_{0}(x), & x \in \mathbb{R}\end{cases}
$$

where $F$ is a smooth function, the constant $\nu>0$, and $U$ and $\partial_{x} U$ satisfy certain decay conditions at infinity. The semidiscrete Hermite function method for (4.11) is to find $u_{N} \in V_{N}$ such that for any $\varphi_{N} \in \mathbb{P}_{N}(\mathbb{R})$,

$$
\left\{\begin{array}{l}
\left(\partial_{t} u_{N}(t), \varphi_{N}\right)+\left(\partial_{x} F\left(u_{N}(t)\right), \varphi_{N}\right)+\nu\left(\partial_{x} u_{N}(t), \partial_{x} \varphi_{N}\right)=\left(f(t), \varphi_{N}\right), \quad t \in(0, T), \\
\left(u_{N}(0), \varphi_{N}\right)=\left(U_{0}, \varphi_{N}\right) .
\end{array}\right.
$$

We investigate the stability property of the scheme (4.12). Suppose that $u_{N}$ and the term on the right-hand side of (4.12) have the errors $\tilde{u}_{N}$ and $\tilde{f}$, respectively. Then, we have

$$
\left(\partial_{t} \tilde{u}_{N}, \varphi_{N}\right)+\left(\partial_{x} \tilde{F}, \varphi_{N}\right)-\nu\left(\partial_{x}^{2} \tilde{u}_{N}, \varphi_{N}\right)=\left(\tilde{f}, \varphi_{N}\right) \quad \forall \varphi_{N} \in \mathbb{P}_{N}(\mathbb{R}), t \in(0, T),
$$

where $\tilde{F}:=F\left(u_{N}+\tilde{u}_{N}\right)-F\left(u_{N}\right)$. Taking $\varphi_{N}=\omega_{\alpha}^{-1} \tilde{u}_{N}$ in (4.13), we obtain, similarly to $(4.8)$,

$$
\begin{aligned}
& \frac{d}{d t}\left\|u_{N}(t)\right\|_{\omega_{\alpha}^{-1}}^{2}+\underline{\delta} \nu\left(\left|u_{N}(t)\right|_{1, \omega_{\alpha}^{-1}}^{2}+\left|\omega_{\alpha}^{-1} u_{N}(t)\right|_{1, \omega_{\alpha}}^{2}\right) \\
& \quad=2\left(\tilde{f}(t)-\partial_{x} \tilde{F}(t), \tilde{u}_{N}(t)\right)_{\omega_{\alpha}^{-1}} \\
& \quad \leq 2(\underline{\delta} \nu)^{-1}\left(\left\|\partial_{x}^{-1} \tilde{f}(t)\right\|_{\omega_{\alpha}^{-1}}^{2}+\|\tilde{F}\|_{\omega_{\alpha}^{-1}}^{2}\right)+\underline{\delta} \nu\left|\omega_{\alpha}^{-1} \tilde{u}_{N}(t)\right|_{1, \omega_{\alpha}}^{2} .
\end{aligned}
$$

Let $\tilde{M}$ be a positive constant and let

$$
M(u)=\max _{0 \leq s \leq T}\left\|u_{N}(s)\right\|_{L^{\infty}(I)}, \quad C_{F}=\max _{|z| \leq M(u)+\tilde{M}}\left|F^{\prime}(z)\right| .
$$


For any given $t \in(0, T)$, if

$$
2\left(\alpha^{2} N\right)^{1 / 4}\left\|\tilde{u}_{N}(s)\right\|_{\omega_{\alpha}^{-1}} \leq \tilde{M} \quad \forall s \in(0, t),
$$

then by (3.15),

$$
\begin{aligned}
& \left\|\tilde{u}_{N}(s)\right\|_{L^{\infty}(I)} \leq \tilde{M} \\
& \|\tilde{F}(s)\|_{\omega_{\alpha}^{-1}}=\left\|\int_{0}^{1} F^{\prime}\left(u_{N}(s)+\theta \tilde{u}_{N}(s)\right) \tilde{u}_{N}(s) d \theta\right\|_{\omega_{\alpha}^{-1}} \leq C_{F}\left\|\tilde{u}_{N}(s)\right\|_{\omega_{\alpha}^{-1}} \quad \forall s \in(0, t) .
\end{aligned}
$$

Substituting the above estimates into (4.14) gives

$$
\frac{d}{d t}\left\|\tilde{u}_{N}(t)\right\|_{\omega_{\alpha}^{-1}}^{2}+\underline{\delta} \nu\left|\tilde{u}_{N}(t)\right|_{1, \omega_{\alpha}^{-1}}^{2} \leq 2(\underline{\delta} \nu)^{-1}\left(C_{F}\left\|\tilde{u}_{N}(t)\right\|_{\omega_{\alpha}^{-1}}^{2}+\left\|\partial_{x}^{-1} \tilde{f}(t)\right\|_{\omega_{\alpha}^{-1}}^{2}\right) .
$$

Define

$$
\begin{gathered}
E\left(\tilde{u}_{N}, t\right)=\left\|\tilde{u}_{N}(t)\right\|_{\omega_{\alpha(t)}^{-1}}^{2}+\underline{\delta} \nu \int_{0}^{t}\left|\tilde{u}_{N}(s)\right|_{1, \omega_{\alpha(s)}^{-1}}^{2} d s, \\
\rho\left(\tilde{u}_{N}, \tilde{f}, t\right)=\left\|\tilde{u}_{N}(0)\right\|_{\omega_{\alpha(0)}^{-1}}^{2}+2(\underline{\delta} \nu)^{-1} \int_{0}^{t}\left\|\partial_{x}^{-1} \tilde{f}(s)\right\|_{\omega_{\alpha(s)}^{-1}}^{2} d s .
\end{gathered}
$$

Integrating (4.16) with respect to $t$ yields

$$
E\left(\tilde{u}_{N}, t\right) \leq \rho\left(\tilde{u}_{N}, \tilde{f}, t\right)+C \int_{0}^{t} E\left(\tilde{u}_{N}, s\right) d s,
$$

where $C$ is a positive constant depending on $(\underline{\delta} \nu)^{-1}$ and $C_{F}$. Then, by a nonlinear Gronwall-like inequality [14],

$$
E\left(\tilde{u}_{N}, t\right) \leq \mathrm{e}^{C t} \rho\left(\tilde{u}_{N}, \tilde{f}, t\right) \quad \forall 0<t \leq T,
$$

provided that

$$
4 \alpha(t) N^{1 / 2} \mathrm{e}^{C t} \rho\left(\tilde{u}_{N}, \tilde{f}, t\right) \leq \tilde{M}^{2} .
$$

We now consider the convergence for the semidiscrete scheme (4.12). As we have shown for the linear problem (1.1), the projection $\mathcal{P}_{N}^{\alpha} U$ is a good comparison function. Let $u_{*}=\mathcal{P}_{N}^{\alpha} U$. Then, for any $\varphi_{N} \in \mathbb{P}_{N}(\mathbb{R})$,

$$
\left\{\begin{array}{l}
\left(\partial_{t} u_{*}(t), \varphi_{N}\right)+\left(\partial_{x} F\left(u_{*}(t)\right), \varphi_{N}\right)+\nu\left(\partial_{x} u_{*}(t), \partial_{x} \varphi_{N}\right)=\left(f(t), \varphi_{N}\right)-\left(\partial_{x} g(t), \varphi_{N}\right), \\
\left(u_{*}(0), \varphi_{N}\right)=\left(U_{0}, \varphi_{N}\right),
\end{array}\right.
$$

where $g(t)=F(U(t))-F\left(u_{*}(t)\right)$. Let $e_{N}=u_{N}-u_{*}$. We have

$$
\left\{\begin{array}{l}
\left(\partial_{t} e_{N}(t), \varphi_{N}\right)+\left(\partial_{x} G(t), \varphi_{N}\right)+\nu\left(\partial_{x} e_{N}(t), \partial_{x} \varphi_{N}\right)=\left(\partial_{x} g(t), \varphi_{N}\right), \quad t \in(0, T), \\
\left(e_{N}(0), \varphi_{N}\right)=0
\end{array}\right.
$$


where $G(t)=F\left(u_{*}(t)+e_{N}(t)\right)-F\left(u_{*}(t)\right)$. Using the same argument as used in deriving the stability result (4.20), we can obtain

$$
\begin{aligned}
\left\|e_{N}(t)\right\|_{\omega_{\alpha(t)}^{-1}}^{2} & \leq C \int_{0}^{t}\|g(s)\|_{\omega_{\alpha(s)}^{-1}}^{2} d s \leq C C_{F}^{\prime} \int_{0}^{t}\left\|\left(I-\mathcal{P}_{N}^{\alpha}\right) U(s)\right\|_{\omega_{\alpha(s)}^{-1}}^{2} d s \\
& \left.\leq C N^{-\sigma} \int_{0}^{t}\left\|\partial_{x}^{\sigma} U(s)\right\|_{\omega_{\alpha(s)}^{-1}}^{2} d s \leq C N^{-\sigma}\|U\|_{L^{2}\left(0, T ; H_{\omega_{\alpha}^{-1}}\right.}^{2}(\mathbb{R})\right) .
\end{aligned}
$$

THEOREM 4.1. Let $U$ and $u_{N}$ be the solutions of (4.11) and (4.12), respectively. Assume that $U \in C\left(0, T ; H_{\omega_{\alpha}^{-1}}^{\sigma}(\mathbb{R})\right)(\sigma \geq 1), F(z) \in C^{1}(\mathbb{R})$, the function $\alpha(t)$ is defined by (2.1), and $\underline{\delta}$ defined by (4.5) is positive. Then

$$
\left\|u_{N}(t)-U(t)\right\|_{\omega_{\alpha}^{-1}} \leq C N^{-\sigma / 2} \quad \forall 0<t<T,
$$

where $C$ is a constant depending on $(\underline{\delta} \nu)^{-1}, \delta_{0}, \delta, T$, and the regularity of $U$ and $F$.

5. Stability and convergence: Fully discrete scheme. In this section, we further discretize the scheme (4.12) by using the method-of-lines approach. Without loss of generality, the analysis will be carried out for the nonlinear convection-diffusion equations. Noting that

$$
\left(\partial_{x} F\left(u_{N}\right), H_{m}^{(\alpha)}\right)=-\sqrt{2 m} \alpha\left(F\left(u_{N}\right), H_{m-1}^{(\alpha)}\right),
$$

we can rewrite the scheme (4.12) in a matrix form as in (4.3):

$$
\frac{d \mathbf{u}}{d t}-\alpha(t) \mathbf{D}^{1 / 2} \mathbf{F}\left(u_{N}\right)+\nu \alpha(t)^{2} \mathbf{B u}=\mathbf{f},
$$

where $\mathbf{D}, \mathbf{B}, \mathbf{f}$ are the same as in (4.4) and (4.5), and the elements of the vector $\mathbf{F}$ are defined by

$$
(\mathbf{F})_{0}=0, \quad(\mathbf{F})_{m}=\left(F\left(u_{N}\right), H_{m-1}^{(\alpha)}\right) \quad(1 \leq m \leq N) .
$$

For the time discretization, we use a second-order Crank-Nicolson/leapfrog scheme, which is implicit for the linear term and explicit for the nonlinear term [14, 15]. For the similarity transformation method (1.5), if the step size $\Delta s$ for the transformed variable $s$ is fixed, then the corresponding time-step in $t$ is nonuniform. In our present approach, a uniform time-step is employed.

Let $\tau$ be the time-step size and let $t_{k}=k \tau\left(k=0,1, \ldots, n_{T} ; T=n_{T} \tau\right)$. We denote $v\left(x, t_{k}\right)$ by $v^{k}(x)$ or simply by $v^{k}$ and $\mathbf{v}\left(t_{k}\right)$ by $\mathbf{v}^{k}$. Let

$$
\mathbf{v}_{\hat{t}}^{k}=\frac{1}{2 \tau}\left(\mathbf{v}^{k+1}-\mathbf{v}^{k-1}\right), \quad \mathbf{v}^{\hat{k}}=\frac{1}{2}\left(\mathbf{v}^{k+1}+\mathbf{v}^{k-1}\right) .
$$

For $v=\omega_{\alpha(t)} \sum_{l=0}^{\infty} \hat{v}_{l}(t) H_{l}^{(\alpha(t))}$, we define

$$
D_{t} v=\omega_{\alpha} \sum_{l=0}^{\infty} \frac{d \hat{v}_{l}}{d t} H_{l}^{(\alpha)} .
$$

The fully discrete Hermite spectral method to the nonlinear convection-diffusion equation (4.11) is to find

$$
u_{N}^{k}=\omega_{\alpha(t)} \sum_{l=0}^{N} \hat{u}_{l}^{k}(t) H_{l}^{(\alpha(t))} \in V_{N}
$$


satisfying

$$
\begin{cases}\mathbf{u}_{\hat{t}}^{k}-\alpha^{k} \mathbf{D}^{1 / 2} \mathbf{F}\left(u_{N}^{k}\right)+\nu\left(\alpha^{k}\right)^{2} \mathbf{B} \mathbf{u}^{\hat{k}}=\mathbf{f}^{k}, & 1 \leq k \leq n_{T}-1, \\ \left(\mathbf{u}^{1}\right)_{m}=\left(\mathbf{u}^{0}\right)_{m}+\tau\left(D_{t} U(0), H_{m}^{(\alpha(0))}\right), & 0 \leq m \leq N, \\ \left(\mathbf{u}^{0}\right)_{m}=\left(U_{0}, H_{m}^{(\alpha(0))}\right), & 0 \leq m \leq N,\end{cases}
$$

where $\left(D_{t} U(0), H_{m}^{(\alpha(0))}\right)$ can be computed from $\frac{d \mathbf{u}}{d t}(0)$ using the initial condition and (5.1).

We now present a stability analysis for the scheme (5.2). Assume that the solution and the term on the right-hand side of (5.2) have errors $\tilde{\mathbf{u}}^{k}:=\left(\tilde{\hat{u}}_{0}^{k}, \tilde{\hat{u}}_{1}^{k}, \ldots, \tilde{\hat{u}}_{N}^{k}\right)^{T}$ and $\tilde{\mathbf{f}}^{k}$, respectively, with $\tilde{u}_{N}^{k}=\omega_{\alpha} \sum_{l=0}^{N} \tilde{\hat{u}}_{l}^{k} H_{l}^{(\alpha)}$. Then the errors satisfy

$$
\tilde{\mathbf{u}}_{\hat{t}}^{k}-\alpha^{k} \mathbf{D}^{1 / 2} \tilde{\mathbf{F}}^{k}+\nu\left(\alpha^{k}\right)^{2} \mathbf{B} \tilde{\mathbf{u}}^{\hat{k}}=\tilde{\mathbf{f}}^{k}, \quad 1 \leq k \leq n_{T}-1,
$$

where $\tilde{\mathbf{F}}^{k}=\mathbf{F}\left(u_{N}^{k}+\tilde{u}_{N}^{k}\right)-\mathbf{F}\left(u_{N}^{k}\right)$. Multiplying both sides of (5.3) with $2 \tilde{\mathbf{u}}^{\hat{k}}$ and assuming that $\underline{\delta}=\min \left\{1,2 \delta_{0} \delta-1\right\}>0$, we obtain

$$
\begin{aligned}
& \left(\left\|\tilde{\mathbf{u}}^{k}\right\|^{2}\right)_{\hat{t}}+2 \underline{\delta} \nu\left(\alpha^{k}\right)^{2}\left\|(\mathbf{D}+\mathbf{I})^{1 / 2} \tilde{\mathbf{u}}^{\hat{k}}\right\|^{2} \leq 2\left(\tilde{\mathbf{f}}^{\hat{k}}+\alpha^{k} \mathbf{D}^{1 / 2} \tilde{\mathbf{F}}^{k}, \tilde{\mathbf{u}}^{\hat{k}}\right) \\
& \quad \leq 2(\underline{\delta} \nu)^{-1}\left(\left(\alpha^{k}\right)^{-2}\left\|(\mathbf{D}+\mathbf{I})^{-1 / 2} \tilde{\mathbf{f}}^{k}\right\|^{2}+\left\|\tilde{\mathbf{F}}^{k}\right\|^{2}\right)+\underline{\delta} \nu\left(\alpha^{k}\right)^{2}\left\|(\mathbf{D}+\mathbf{I})^{1 / 2} \tilde{\mathbf{u}}^{\hat{k}}\right\|^{2} .
\end{aligned}
$$

Let $\tilde{M}$ be a positive constant and let

$$
M(u)=\max _{0 \leq k \leq n_{T}}\left\|u_{N}^{k}\right\|_{L^{\infty}(I)}, \quad C_{F}=\max _{|z| \leq M(u)+\tilde{M}}\left|F^{\prime}(z)\right| .
$$

For a fixed $n \leq n_{T}$, if

$$
\left\|\tilde{\mathbf{u}}^{k}\right\|=\left\|\tilde{u}_{N}^{k}\right\|_{\omega_{\alpha}^{-1}} \leq\left(4 \alpha^{k} N^{1 / 2}\right)^{-1 / 2} \tilde{M} \quad \forall 1 \leq k \leq n-1,
$$

then, by (3.15), we have $\left\|\tilde{u}_{N}^{k}\right\|_{L^{\infty}(I)} \leq \tilde{M}$ and

$$
\begin{aligned}
\left\|\tilde{\mathbf{F}}^{k}\right\| & =\left\|\mathcal{P}_{N-1}^{\alpha}\left(F\left(u_{N}^{k}+\tilde{u}_{N}^{k}\right)-F\left(u_{N}^{k}\right)\right)\right\|_{\omega_{\alpha}^{-1}} \\
& \leq\left\|F\left(u_{N}^{k}+\tilde{u}_{N}^{k}\right)-F\left(u_{N}^{k}\right)\right\|_{\omega_{\alpha}^{-1}} \leq C_{F}\left\|\tilde{u}_{N}^{k}\right\|_{\omega_{\alpha}^{-1}}^{2}=C_{F}\left\|\tilde{\mathbf{u}}^{k}\right\| .
\end{aligned}
$$

Define

$$
\begin{aligned}
& E^{n}(\mathbf{v})=\left\|\mathbf{v}^{n}\right\|^{2}+2 \underline{\delta} \nu \tau \sum_{k=1}^{n-1}\left(\alpha^{k}\right)^{2}\left\|(\mathbf{D}+\mathbf{I})^{1 / 2} \mathbf{v}^{\hat{k}}\right\|^{2}, \\
& \rho^{n}(\mathbf{v}, \mathbf{g})=\left\|\mathbf{v}^{0}\right\|^{2}+\left\|\mathbf{v}^{1}\right\|^{2}+4(\underline{\delta} \nu)^{-1} \tau \sum_{k=0}^{n-1}\left(\alpha^{k}\right)^{-2}\left\|(\mathbf{D}+\mathbf{I})^{-1 / 2} \mathbf{g}^{k}\right\|^{2} .
\end{aligned}
$$

Summing (5.4) for $1 \leq k \leq n-1$ gives

$$
E^{n}(\tilde{\mathbf{u}}) \leq \rho^{n}(\tilde{\mathbf{u}}, \tilde{\mathbf{f}})+2(\underline{\delta} \nu)^{-1} C_{F} \tau \sum_{k=1}^{n-1} E^{k}(\tilde{\mathbf{u}}) .
$$

It follows from a discrete nonlinear Gronwall-like inequality [14] that

$$
E^{n}(\tilde{\mathbf{u}}) \leq \mathrm{e}^{C n \tau} \rho^{n}(\tilde{\mathbf{u}}, \tilde{\mathbf{f}}) \quad \forall 0<n \leq n_{T},
$$

provided that $4 \max _{0 \leq k \leq n} \alpha^{k} N^{1 / 2} \mathrm{e}^{C k \tau} \rho^{k}(\tilde{\mathbf{u}}, \tilde{\mathbf{f}}) \leq \tilde{M}^{2}$.

THEOREM 5.1. Let $u_{N}$ be the solution of (4.12) and let $\tilde{M}$ be a positive number. Assume that the function $\alpha(t)$ is defined by (2.1) and that $\underline{\delta}$ defined by (4.5) is positive. For $0<n \leq n_{T}$, if 


$$
4 \max _{0 \leq k \leq n} \alpha^{k} N^{1 / 2} \mathrm{e}^{C k \tau} \rho^{k}(\tilde{\mathbf{u}}, \tilde{\mathbf{f}}) \leq \tilde{M}^{2},
$$

then

$$
E^{k}(\tilde{\mathbf{u}}) \leq \mathrm{e}^{C k \tau} \rho^{k}(\tilde{\mathbf{u}}, \tilde{\mathbf{f}}) \quad \forall 0<k \leq n,
$$

where $E^{k}$ and $\rho^{k}$ are defined by (5.6) and (5.7), respectively, and $C$ is a constant linearly proportional to $(\underline{\delta} \nu)^{-1}$ and $C_{F}$.

We now analyze the convergence of the fully discrete scheme (5.2). Let $u_{*}=\mathcal{P}_{N}^{\alpha} U$ with the following Hermite expansion:

$$
u_{*}=\omega_{\alpha} \sum_{l=0}^{N} \hat{u}_{* l} H_{l}^{(\alpha)}
$$

Denote the coefficients of the above expansion by $\mathbf{u}_{*}:=\left(\hat{u}_{* 0}, \hat{u}_{* 1}, \ldots, \hat{u}_{* N}\right)^{T}$. It follows from (4.22) that

$$
\mathbf{u}_{* \hat{t}}^{k}-\alpha^{k} \mathbf{D}^{1 / 2} \mathbf{F}\left(u_{*}^{k}\right)+\nu\left(\alpha^{k}\right)^{2} \mathbf{B} \mathbf{u}_{*}^{\hat{k}}=\mathbf{f}^{k}-\mathbf{g}^{k},
$$

where we split $\mathbf{g}^{k}$ into $\mathbf{g}_{1}^{k}, \mathbf{g}_{2}^{k}, \mathbf{g}_{3}^{k}$ as follows:

$$
\begin{aligned}
\mathbf{g}^{k} & =\left[\left(\frac{d \mathbf{u}_{*}}{d t}\right)^{k}-\mathbf{u}_{* \hat{t}}^{k}\right]+\left[\alpha^{k} \mathbf{D}^{1 / 2}\left(\mathbf{F}\left(u_{*}^{k}\right)-\mathbf{F}\left(U^{k}\right)\right)\right]+\left[\nu\left(\alpha^{k}\right)^{2} \mathbf{B}\left(\mathbf{u}_{*}^{k}-\mathbf{u}_{*}^{\hat{k}}\right)\right] \\
& =: \mathbf{g}_{1}^{k}+\mathbf{g}_{2}^{k}+\mathbf{g}_{3}^{k}
\end{aligned}
$$

Let $e_{N}^{k}=u_{N}^{k}-u_{*}^{k}$ and $\mathbf{e}^{k}=\mathbf{u}^{k}-\mathbf{u}_{*}^{k}$. Then it can be verified that

$$
\left\{\begin{array}{l}
\mathbf{e}_{\hat{t}}^{k}-\alpha^{k} \mathbf{D}^{1 / 2} \mathbf{G}^{k}+\nu\left(\alpha^{k}\right)^{2} \mathbf{B} \mathbf{e}^{\hat{k}}=\mathbf{g}^{k}, \quad 1 \leq k \leq n_{T}-1, \\
\mathbf{e}^{0}=0, \quad \mathbf{e}^{1}=\mathbf{u}_{*}(0)+\tau \frac{d \mathbf{u}_{*}}{d t}(0)-\mathbf{u}_{*}(\tau),
\end{array}\right.
$$

where $\mathbf{G}^{k}=\mathbf{F}\left(u_{*}^{k}+e_{N}^{k}\right)-\mathbf{F}\left(u_{*}^{k}\right)$. By the same arguments as in the stability analysis above, we can obtain

$$
\left\|e_{N}^{n}\right\|_{\omega_{\alpha}^{-1}}^{2}=\left\|\mathbf{e}^{n}\right\|^{2} \leq C\left(\left\|\mathbf{e}^{0}\right\|^{2}+\left\|\mathbf{e}^{1}\right\|^{2}+(\underline{\delta} \nu)^{-1} \tau \sum_{k=0}^{n-1}\left(\alpha^{k}\right)^{-2}\left\|(\mathbf{D}+\mathbf{I})^{-1 / 2} \mathbf{g}^{k}\right\|^{2}\right) .
$$

The last term on the right-hand side can be bounded by using the facts below:

$$
\begin{aligned}
\tau \sum_{k=0}^{n-1}\left\|(\mathbf{D}+\mathbf{I})^{-1 / 2} \mathbf{g}_{1}^{k}\right\|^{2} & \leq C \tau^{4}\left\|D_{t}^{3} U\right\|_{L^{2}\left(0, T ; H_{\omega_{\alpha}^{-1}}^{-1}(\mathbb{R})\right)}^{2} \\
\tau \sum_{k=0}^{n-1}\left\|(\mathbf{D}+\mathbf{I})^{-1 / 2} \mathbf{g}_{2}^{k}\right\|^{2} & \leq C \tau \sum_{k=0}^{n-1}\left\|F\left(u_{*}^{k}\right)-F\left(U^{k}\right)\right\|_{\omega_{\alpha}^{-1}}^{2} \\
& \leq C C_{F}^{\prime} N^{-\sigma}\|U\|_{C\left(0, T ; H_{\omega_{\alpha}^{-1}}^{\sigma}(\mathbb{R})\right)}^{2}, \\
\tau \sum_{k=0}^{n-1}\left\|(\mathbf{D}+\mathbf{I})^{-1 / 2} \mathbf{g}_{3}^{k}\right\|^{2} & \leq C \tau^{4}\left\|D_{t}^{2} U\right\|_{L^{2}\left(0, T ; H_{\omega_{\alpha}^{-1}}^{1}(\mathbb{R})\right)}^{2}
\end{aligned}
$$


The initial errors can be bounded by using the Taylor expansion:

$$
\begin{aligned}
\left\|\mathbf{e}^{1}\right\| & =\left\|\int_{0}^{\tau}(\tau-s) \frac{d^{2} \mathbf{u}_{*}}{d t^{2}}(s) d s\right\| \leq \tau^{2} \max _{0 \leq s \leq \tau}\left\|\frac{d^{2} \mathbf{u}_{*}}{d t^{2}}(s)\right\| \\
& \left.\leq \tau^{2}\left\|D_{t}^{2} U(s)\right\|_{C\left(0, \tau ; L_{\omega_{\alpha}^{-1}}{ }^{-1}\right.}(\mathbb{R})\right) .
\end{aligned}
$$

Combining the above results, we arrive at the following optimal error estimate.

THEOREM 5.2. Let $U$ and $u_{N}$ be the solutions of (4.11) and (4.12), respectively. Assume that $U \in C\left(0, T ; H_{\omega_{\alpha}^{-1}}^{\sigma}(\mathbb{R})\right)(\sigma \geq 1), D_{t}^{2} U \in L^{2}\left(0, T ; H_{\omega_{\alpha}^{-1}}^{1}(\mathbb{R})\right) \cap$ $C\left(0, \tau ; L_{\omega_{\alpha}^{-1}}^{2}(\mathbb{R})\right), D_{t}^{3} U \in L^{2}\left(0, T ; H_{\omega_{\alpha}^{-1}}^{-1}(\mathbb{R})\right)$, and $F(z) \in C^{1}(\mathbb{R})$. Moreover, assume that the function $\alpha(t)$ is defined by (2.1), $\underline{\delta}$ defined by (4.5) is positive, and $\tau N^{1 / 8} \leq c_{0}$ is sufficiently small. Then, for $0 \leq n \leq n_{T}$,

$$
\left\|u_{N}^{n}-U^{n}\right\|_{\omega_{\alpha\left(t_{n}\right)}^{-1}} \leq C\left(\tau^{2}+N^{-\sigma / 2}\right)
$$

where $C$ is a constant depending on $(\underline{\delta} \nu)^{-1}, \delta_{0}, \delta, T$, and the regularity of $U$ and $F$.

Remark 5.1. If the underlying PDE solution does not satisfy the exponential decay property required by the Hermite function approximation, one may use the Hermite polynomial approximation directly. In this case, the Hermite polynomial approximation should be used together with a time-dependent scaling,

$$
\alpha(t)=\frac{1}{2 \sqrt{\nu \delta_{0}(\delta(T-t)+1)}} .
$$

For the linear parabolic equation (1.1) and the nonlinear convection-diffusion equation (4.11), it can be verified that with the choice (5.16), the desired stability and convergence results can be established in some appropriate function space.

6. Numerical results. In this section, we present some numerical examples using the proposed method for both linear and nonlinear equations. The numerical results will be compared with those obtained by using the classical method (1.3) and by using the similarity transformation technique. In the following computations, the integrals involved are computed by the Hermite-Gauss quadrature rules with $N+1$ quadrature points. Let

$$
E_{N}(t)=\left\|u_{N}(t)-U^{N}(t)\right\|_{\omega_{\alpha}^{-1}}, \quad E_{N, \infty}(t)=\frac{\max _{0 \leq j \leq N}\left|u_{N}\left(y_{j}, t\right)-U\left(y_{j}, t\right)\right|}{\max _{0 \leq j \leq N}\left|U\left(y_{j}, t\right)\right|},
$$

where $U^{N} \in V_{N}$ is the interpolation of $U$ at the Hermite-Gauss points $\left\{y_{j}\right\}_{j=0}^{N}$. The examples used below are taken from [6] and [10], where the diffusion coefficient $\nu$ in (1.1) is chosen as 1 . In the linear case our approach is appropriate for the general choice of $\nu>0$ due to the use of the scaling factor (2.1). However, for nonlinear problems (such as Example 6.3 below) with sufficiently small values of $\nu$, steep layers may be developed, and in this case some special techniques such as the spectral viscosity method [16] should be applied.

Example 6.1 (linear problem). Consider the parabolic problem (1.1) with $\nu=1$ and the following source term:

$$
f(x, t)=(x \cos x+(t+1) \sin x)(t+1)^{-3 / 2} e^{-x^{2} / 4(t+1)} .
$$


TABle 6.1

Example 6.1: Errors at $t=1$ with $N=20$ using different methods.

\begin{tabular}{|c|c|c|c|}
\hline $\begin{array}{c}\text { Time step } \\
\tau\end{array}$ & $\begin{array}{c}\text { Funaro and Kavian's } \\
\text { scheme [6] }\end{array}$ & $\begin{array}{c}\text { Classical } \\
\text { scheme (1.3) }\end{array}$ & $\begin{array}{c}\text { Proposed } \\
\text { scheme }(2.7)\end{array}$ \\
\hline $250^{-1}$ & $2.487 \mathrm{E}-03$ & $1.948 \mathrm{E}-04$ & $2.958 \mathrm{E}-06$ \\
\hline $1000^{-1}$ & $6.203 \mathrm{E}-04$ & $1.947 \mathrm{E}-04$ & $1.189 \mathrm{E}-06$ \\
\hline $4000^{-1}$ & $1.550 \mathrm{E}-04$ & $1.947 \mathrm{E}-04$ & $1.177 \mathrm{E}-06$ \\
\hline $16000^{-1}$ & $3.886 \mathrm{E}-05$ & $1.947 \mathrm{E}-04$ & $1.177 \mathrm{E}-06$ \\
\hline
\end{tabular}

TABLE 6.2

Example 6.1: Errors of the proposed scheme (2.7) with different $\tau$ and $N$.

\begin{tabular}{|r|c|c|c|c|}
\hline$\tau$ & $N$ & $E_{N}(1)$ & $E_{N, \infty}(1)$ & Order \\
\hline $1 \mathrm{E}-1$ & & $1.697 \mathrm{E}-03$ & $9.775 \mathrm{E}-04$ & \\
$1 \mathrm{E}-2$ & & $1.697 \mathrm{E}-05$ & $9.769 \mathrm{E}-06$ & $\tau^{2.00}$ \\
$1 \mathrm{E}-3$ & \multirow{2}{*}{30} & $1.696 \mathrm{E}-07$ & $9.769 \mathrm{E}-08$ & $\tau^{2.00}$ \\
$1 \mathrm{E}-4$ & & $1.696 \mathrm{E}-09$ & $9.798 \mathrm{E}-10$ & $\tau^{2.00}$ \\
\hline \hline & 10 & $5.161 \mathrm{E}-03$ & $1.192 \mathrm{E}-03$ & \\
$1 \mathrm{E}-4$ & 20 & $1.177 \mathrm{E}-06$ & $1.246 \mathrm{E}-07$ & $N^{-12.10}$ \\
& 30 & $1.696 \mathrm{E}-09$ & $9.798 \mathrm{E}-10$ & $N^{-16.14}$ \\
\hline
\end{tabular}

This example was used by Funaro and Kavian [6]. Its exact solution is of the form

$$
U(x, t)=\frac{\sin x}{\sqrt{t+1}} e^{-x^{2} / 4(t+1)} .
$$

We solve the above problem with $\left(\delta_{0}, \delta\right)=(1.5,0)$, which corresponds to the classical approach (1.3), and with $\left(\delta_{0}, \delta\right)=(1,1)$, which corresponds to the method proposed in this work. For ease of comparison, we use the same mesh size as used in [6]. Table 6.1 shows the error $E_{20}(t)$ at $t=1$ with different time-steps. Note that the result in [6] is obtained by using (explicit) first-order forward difference in time.

Table 6.2 shows the order of accuracy for the scheme (2.7) with $\delta_{0}=\delta=1$. The numerical results are in good agreement with the theoretical prediction that the numerical scheme (2.7) is of second-order accuracy in time and spectral accuracy in space.

Example 6.2 (linear problem). Consider the parabolic problem (1.1) with $\nu=1$ and the following source term:

$$
f(x, t)=\left(k\left(1+4 c^{2} x\right) \cos k(x+t)-\left(k^{2}+2 c^{2}\left(1-2(c x)^{2}\right)\right) \sin k(x+t)\right) \mathrm{e}^{-(c x)^{2}},
$$

where $c$ is a constant. The exact solution of this example has a time-independent decay:

$$
U(x, t)=\sin k(x+t) \mathrm{e}^{-c^{2} x^{2}} .
$$


TABLE 6.3

Example 6.2: Comparison of the classical approach and the present method.

\begin{tabular}{|c|r|c|c|c|c|}
\hline \multirow{4}{*}{$\tau$} & \multirow{4}{*}{$1 \mathrm{E}-3$} & \multicolumn{2}{|c|}{ Classical method (1.3) } & \multicolumn{2}{c|}{ Proposed method (2.7) } \\
\cline { 3 - 6 } & 250 & $5.66 \mathrm{E}-07$ & $3.93 \mathrm{E}-07$ & $4.30 \mathrm{E}-06$ & $1.87 \mathrm{E}-06$ \\
& 500 & $1.52 \mathrm{E}-04$ & $8.50 \mathrm{E}-06$ & $2.73 \mathrm{E}-06$ & $2.03 \mathrm{E}-06$ \\
& 750 & $3.72 \mathrm{E}+01$ & $2.67 \mathrm{E}+00$ & $2.08 \mathrm{E}-06$ & $1.44 \mathrm{E}-06$ \\
& 1000 & $8.20 \mathrm{E}+06$ & $2.02 \mathrm{E}+05$ & $1.75 \mathrm{E}-06$ & $1.34 \mathrm{E}-06$ \\
\hline \multirow{5}{*}{$1 \mathrm{E}-4$} & 2500 & $5.66 \mathrm{E}-09$ & $3.94 \mathrm{E}-09$ & $4.30 \mathrm{E}-08$ & $1.87 \mathrm{E}-08$ \\
& 5000 & $1.45 \mathrm{E}-04$ & $8.01 \mathrm{E}-06$ & $2.73 \mathrm{E}-08$ & $2.03 \mathrm{E}-08$ \\
& 7500 & $4.30 \mathrm{E}+01$ & $2.41 \mathrm{E}+00$ & $2.08 \mathrm{E}-08$ & $1.44 \mathrm{E}-08$ \\
& 10000 & $8.95 \mathrm{E}+06$ & $4.97 \mathrm{E}+04$ & $1.73 \mathrm{E}-08$ & $1.36 \mathrm{E}-08$ \\
\hline
\end{tabular}

The purpose for choosing this example is to demonstrate that the Hermite spectral method with a time-dependent scaling also works well for the solutions with timeindependent decays. In our computations, the parameters $k$ and $c$ are taken as 5 and 0.5 , respectively. We solve this problem by using a constant weight $\alpha(t) \equiv$ 0.5 , which not only corresponds to the classical method (1.3) but also matches the exponential solution-decay exactly. We also solve the problem by using the scheme (2.7) with $\left(\delta_{0}, \delta\right)=(0.6,1)$. This choice of the parameters satisfies $\underline{\delta}=0.2>0$, and therefore stability and convergence are expected. It is seen from Table 6.3 that although the classical method (1.3) matches the exponential decay exactly, the error is accumulated due to numerical instability. On the other hand, the Hermite spectral method with a time-dependent scaling produces highly accurate and stable numerical approximations.

Example 6.3 (nonlinear viscous Burgers equation). Consider the viscous Burgers equation

$$
\partial_{t} U+U \partial_{x} U-\nu \partial_{x}^{2} U=f(x, t), \quad x \in \mathbb{R}, \quad t>0 .
$$

It was computed in [10] via the transformation

$$
y=\frac{x}{2 \sqrt{\nu(t+1)}}, \quad s=\ln (t+1)
$$

for a soliton-like solution

$$
U(x, t)=\mathrm{e}^{-y^{2}} \operatorname{sech}^{2}(a y-b s-c) .
$$

We will recompute this problem with parameters $a=0.3, b=0.5, c=-3$, and $\nu=1$.

We use the fully discrete scheme (5.2) to solve the problem with $\left(\delta_{0}, \delta\right)=(1,1)$. The numerical errors at $t=\mathrm{e}-1$ are presented in Table 6.4, where the comparison is made with those given in [10]. It is seen that the present method is more accurate than the similarity transformation solution.

To show the rate of convergence for (5.2), we list in Table 6.5 the numerical errors at $t=1$ with various $\tau$ and $N$. The fully discrete scheme (5.2) is applied to the viscous Burgers problem with $\left(\delta_{0}, \delta\right)=(1,1)$. It again confirms the theoretical prediction that the present method is of second-order accuracy in time and spectral accuracy in space. 
TABLE 6.4

Example 6.3: Errors at $t=\mathrm{e}-1$ with $\tau=0.001 * t$.

\begin{tabular}{|c|c|c|}
\hline$N$ & Guo and Xu's result $[10]$ & Proposed scheme (5.2) \\
\hline 8 & $1.381 \mathrm{E}-06$ & $1.563 \mathrm{E}-05$ \\
\hline 16 & $1.381 \mathrm{E}-06$ & $6.337 \mathrm{E}-07$ \\
\hline 32 & $1.381 \mathrm{E}-06$ & $1.031 \mathrm{E}-07$ \\
\hline
\end{tabular}

TABLE 6.5

Example 6.3: Errors of the proposed scheme (5.2) with different $\tau$ and $N$.

\begin{tabular}{|c|c|c|c|c|}
\hline$\tau$ & $N$ & $E_{N}(1)$ & $E_{N, \infty}(1)$ & Order \\
\hline $1 \mathrm{E}-1$ & & $5.101 \mathrm{E}-04$ & $4.677 \mathrm{E}-03$ & \\
$1 \mathrm{E}-2$ & & $4.508 \mathrm{E}-06$ & $4.548 \mathrm{E}-05$ & $\tau^{2.05}$ \\
$1 \mathrm{E}-3$ & 40 & $4.454 \mathrm{E}-08$ & $4.530 \mathrm{E}-07$ & $\tau^{2.01}$ \\
$1 \mathrm{E}-4$ & & $4.467 \mathrm{E}-10$ & $4.372 \mathrm{E}-09$ & $\tau^{2.00}$ \\
\hline \hline & 8 & $6.685 \mathrm{E}-06$ & $1.163 \mathrm{E}-04$ & \\
$1 \mathrm{E}-4$ & 16 & $2.684 \mathrm{E}-07$ & $3.121 \mathrm{E}-06$ & $N^{-4.64}$ \\
& 32 & $7.888 \mathrm{E}-10$ & $7.120 \mathrm{E}-09$ & $N^{-8.41}$ \\
\hline
\end{tabular}

Acknowledgment. The third author thanks Prof. David Gottlieb for helpful discussions on numerical approximations in unbounded domains.

\section{REFERENCES}

[1] N. ADžIć, Modified Hermite polynomials in the spectral approximation for boundary layer problems, Bull. Austral. Math. Soc., 45 (1992), pp. 267-276.

[2] C. Bernardi and Y. Maday, Spectral methods, in Handbook of Numerical Analysis, Vol. V, Handb. Numer. Anal. V, P. G. Ciarlet and J. L. Lions, eds., North-Holland, Amsterdam, 1997, pp. 209-485.

[3] J. P. BoyD, Spectral method using rational basis functions on an infinite interval, J. Comput. Phys., 69 (1987), pp. 112-142.

[4] O. Coulaud, D. Funaro, and O. Kavian, Laguerre spectral approximation of elliptic problems in exterior domains, in Spectral and High Order Methods for Partial Differential Equations (Como, 1989), Comput. Methods Appl. Mech. Engrg., 80 (1990), pp. 451-458.

[5] J. C. M. FOK, B. GuO, AND T. TANG, Combined Hermite spectral-finite difference method for the Fokker-Planck equation, Math. Comp., 71 (2002), pp. 1497-1528.

[6] D. Funaro and O. Kavian, Approximation of some diffusion evolution equations in unbounded domains by Hermite functions, Math. Comp., 57 (1991), pp. 597-619.

[7] B.-Y. Guo, Error estimation of Hermite spectral method for nonlinear partial differential equations, Math. Comp., 68 (1999), pp. 1067-1078.

[8] B.-Y. Guo And J. Shen, Laguerre-Galerkin method for nonlinear partial differential equations on a semi-infinite interval, Numer. Math., 86 (2000), pp. 635-654.

[9] B.-Y. Guo, J. Shen, And Z.-Q. WANG, A rational approximation and its applications to differential equations on the half line, J. Sci. Comput., 15 (2000), pp. 117-147.

[10] B.-Y. GuO AND C.-L. XU, Hermite pseudospectral method for nonlinear partial differential equations, M2AN Math. Model. Numer. Anal., 34 (2000), pp. 859-872.

[11] V. Iranzo and A. FAlquÉs, Some spectral approximations for differential equations in unbounded domains, Comput. Methods Appl. Mech. Engrg., 98 (1992), pp. 105-126.

[12] I. K. Khabibrakhmanov and D. Summers, The use of generalized Laguerre polynomials in spectral methods for nonlinear differential equations, Comput. Math. Appl., 36 (1998), pp. $65-70$. 
[13] Y. LiU, L. LiU, AND T. TANG, The numerical computation of connecting orbits in dynamical systems: A rational spectral approach, J. Comput. Phys., 111 (1994), pp. 373-380.

[14] H. Ma AND W. Sun, Optimal error estimates of the Legendre-Petrov-Galerkin method for the Korteweg-de Vries equation, SIAM J. Numer. Anal., 39 (2001), pp. 1380-1394.

[15] H. Ma And W. Sun, A Legendre-Petrov-Galerkin and Chebyshev collocation method for thirdorder differential equations, SIAM J. Numer. Anal., 38 (2000), pp. 1425-1438.

[16] Y. MAdAY AND E. TADMOR, Analysis of the spectral vanishing viscosity method for periodic conservation laws, SIAM J. Numer. Anal., 26 (1989), pp. 854-870.

[17] Y. Maday, B. Pernaud-Thomas, and H. Vandeven, Reappraisal of Laguerre type spectral methods, Rech. Aérospat., 6 (1985), pp. 353-375.

[18] G. Mastroianni and G. Monegato, Nyström interpolants based on zeros of Laguerre polynomials for some Weiner-Hopf equations, IMA J. Numer. Anal., 17 (1997), pp. 621-642.

[19] B. Muckenhoupt, Mean convergence of Hermite and Laguerre series, II, Trans. Amer. Math. Soc., 147 (1970), pp. 433-460.

[20] J. W. Schumer and J. P. Holloway, Vlasov simulations using velocity-scaled Hermite representations, J. Comput. Phys., 144 (1998), pp. 626-661.

[21] J. Shen, Stable and efficient spectral methods in unbounded domains using Laguerre functions, SIAM J. Numer. Anal., 38 (2000), pp. 1113-1133.

[22] T. TANG, The Hermite spectral method for Gaussian-type functions, SIAM J. Sci. Comput., 14 (1993), pp. 594-606.

[23] S. Thangavelu, Hermite expansions on $\mathbf{R}^{2 \mathrm{n}}$ for radial functions, Rev. Mat. Iberoamericana, 6 (1990), pp. 61-73.

[24] S. Thangavelu, Lectures on Hermite and Laguerre Expansions, Princeton University Press, Princeton, NJ, 1993.

[25] J. A. C. Weideman, The eigenvalues of Hermite and rational spectral differentiation matrices, Numer. Math., 61 (1992), pp. 409-432. 